'Departamento de Nefrología, Facultad de Medicina, Escuela de Medicina Pontificia Universidad Católica de Chile. Santiago, Chile. anterno de Medicina, Facultad de Medicina Pontificia Universidad Católica de Chile. Santiago, Chile. bResidente de Medicina Interna, Facultad de Medicina Pontificia Universidad Católica de Chile. Santiago, Chile.

Los autores declaran que no hubo aportes financieros externos en la realización de esta revisión, ni conflictos de interés.

Recibido el 19 de diciembre de 2017, aceptado el 8 de marzo de 2018.

Correspondencia a: Dr. Rodrigo A. Sepúlveda Departamento de Nefrología Diagonal Paraguay 362, piso 4 . Santiago, Chile.

Teléfono: + 56-2-3543229 rrsepulveda@uc.c

\section{Síndrome hemolítico urémico atípico en tratamiento con eculizumab. Casos clínicos}

\author{
RODRIGO TAGLE ${ }^{1}$, GUSTAVO RIVERA ${ }^{\mathrm{a}}$, \\ BENJAMÍN WALBAUM ${ }^{\mathrm{b}}$, RODRIGO A. SEPÚLVEDA ${ }^{1}$
}

\section{Atypical hemolytic uremic syndrome. Report of two cases treated with Eculizumab}

Hemolytic uremic syndrome (HUS) is a type of thrombotic microangiopathy where organic damage predominates in the kidney. Atypical HUS (aHUS) is a rare disease that affects young adults and causes terminal chronic renal failure ending in dialysis, in most cases. It also recurs after kidney transplantation. aHUS is associated with genetic defects of the alternative complement pathway or its activation by other factors such as drugs, autoimmune diseases, infections, malignant hypertension and ischemia-reperfusion. We report two women aged 17 and 25 years old with catastrophic aHUS. In both cases, complement amplifying factors (drugs and infections) were added and acted on a genetic vulnerability to precipitate complement activation and produce aHUS. Both patients developed terminal renal failure and had to undergo hemodialysis. Fortunately, after a broad etiological study, it was possible to make the diagnosis of aHUS and start treatment with Eculizumab, a monoclonal antibody that changed the natural history of aHUS. It inhibits complement activity controlling microangiopathy and preventing the development of end-stage renal disease. It also improves the success rate in kidney transplantation. In the case of our patients, both discontinued dialysis after chronic treatment with Eculizumab.

(Rev Med Chile 2018; 146: 254-259)

Key words: Antibodies, Monoclonal, Humanized; Atypical Hemolytic Uremic Syndrome; Thrombotic Microangiopathies.
E 1 síndrome hemolítico urémico (SHU) es un tipo de microangiopatía trombótica (MAT), donde la afectación orgánica predominante es a nivel renal ${ }^{1-4}$. La activación hemostática se genera en la microvasculatura del parénquima renal provocando trombosis, consumo plaquetario y falla renal. Los eritrocitos circulantes serán traumatizados provocando liberación de lactato deshidrogenasa (LDH) y hemoglobina; hemoglobinuria y consumo de haptoglobina. Se establece una anemia hemolítica microangiopática.

Son múltiples las enfermedades que pueden causar SHU; como infección por Escherichia coli productor de toxina shiga, infección por agentes productores de neuraminidasa (Streptococcus pneumoniae y virus influenza), enfermedades autoinmunes, neoplasias, drogas (quimioterapias, anticalcineurínicos, inhibidores mTOR) y alteraciones genéticas o adquiridas de la vía alterna del complemento ${ }^{1,3}$.

En el SHU atípico (SHUa) la causa de MAT es una activación anómala de la vía alterna del complemento. Esta activación puede ser secundaria a patologías que exacerban la actividad del complemento (infecciones, pancreatitis, embarazo/ puerperio, hipertensión maligna, enfermedades 
autoinmunes, drogas, isquemia, trasplante de órganos, neoplasias) y/o alteraciones genéticas en algún componente de la vía alterna del complemento ${ }^{1,3}$. Este último cuadro se denomina SHUa primario. Para que se desarrolle un SHUa se combinan factores amplificadores del complemento sobre una vulnerabilidad genética; así, cuando se supera un "umbral", se desencadenará la MAT.

Eculizumab es un anticuerpo monoclonal humanizado recombinante contra la fracción C'5 del complemento. Impide la formación de anafilotoxinas y el complejo de ataque de membrana. Es un híbrido IgG2/IgG4 para evitar la activación de macrófagos y el complemento. Ha sido aprobado por la FDA como tratamiento de primera línea en Hemoglobinuria paroxística nocturna y SHUa. El tratamiento puede ser de por vida y el costo anual de éste son 500.000 dólares constituyéndose como el fármaco más caro del mundo.

\section{Caso 1}

Mujer de 17 años con antecedentes de SHUa diagnosticado a los 3 años. En aquella oportunidad presentó una MAT con falla renal que requirió terapia de reemplazo renal (TRR). Se descartó púrpura trombocitopénico trombótico (PTT) con ADAMTS13 74\% (actividad normal: 41\%-180\%) y SHU típico con PCR negativa para Escherichia coli productora de toxina Shiga en deposiciones. La MAT revierte espontáneamente y recupera función renal. A los 6 años tuvo una recurrencia asociada a infección por Mycoplasma pneumoniae sin necesidad de TRR. A los 13 años su creatinina sérica ( $\mathrm{sCr}$ ) era $0,61 \mathrm{mg} / \mathrm{dL}$. No hay otros antecedentes relevantes.

El cuadro actual inicia con cefalea y compromiso del estado general. Evoluciona con sensación febril, coluria, dolor abdominal cólico y deposiciones blandas, sin elementos patológicos; por lo que decide consultar. Se objetiva ictericia y púrpura petequial en extremidades inferiores, sin compromiso neurológico. Laboratorio: hematocrito $31 \%$, hemoglobina $10,9 \mathrm{~g} / \mathrm{dL}$, plaquetas $9.000 / \mu \mathrm{L}$, esquistocitos presentes, bilirrubina indirecta 2,34 $\mathrm{mg} / \mathrm{dL}$ y sCr $1,76 \mathrm{mg} / \mathrm{dL}$.

Evoluciona con mayor deterioro hematológico y renal, requiriendo hemodiálisis (HD). Se plantea diagnóstico de PTT versus SHU; inicia metilprednisolona y plasmaféresis, sin respuesta.
Posteriormente es trasladada a nuestro centro para continuar manejo. Respecto al estudio etiológico: cultivos microbiológicos (-), Coombs directo e indirecto (-), haptoglobina $<6,8 \mathrm{mg} /$ dL (normal: 30-200 mg/dL), ADAMTS13 128\%, $\beta$-HCG negativa, serología VIH, VHC y VHB (-), estudio inmunológico (ANA, ANCA, crioglobulinas, anticuerpos antifosfolípidos, anti- $\beta 2$-glicoproteína-1 y anticoagulante lúpico) negativos, C’3 $83 \mathrm{mg} / \mathrm{dL}$ (normal: $70-150 \mathrm{mg} / \mathrm{dL}$ ) y C'4 $11 \mathrm{mg} /$ dL (normal: 13,5-45 mg/dL). Ecografía doppler renal informa aumento de ecogenicidad cortical con pérdida de diferenciación corticomedular, sin otras alteraciones.

Por necesidad de tratamiento con Eculizumab se administra vacuna contra Neisseria meningitidis y Streptococcus pneumoniae. Tras inicio del fármaco logra remisión hematológica y lenta recuperación de función renal. Es dada de alta en $\mathrm{HD}$, logrando suspensión de ésta al $2^{\circ}$ mes de terapia ( $\mathrm{sCr} 1,5 \mathrm{mg} / \mathrm{dL})$.

Se realizó estudio genético en Alemania, resultando negativo. Debido a que la paciente presentaba SHUa sin mutación demostrable se discontinúa terapia con Eculizumab luego de 6 meses de tratamiento. Sin embargo, 3 meses después presenta recaída, asociada a infección respiratoria viral. La sCr asciende hasta $2,79 \mathrm{mg} /$ $\mathrm{dL}$, pero recupera prontamente su valor basal al administrar Eculizumab. En esta oportunidad se descartó presencia de anticuerpos Anti-Factor $\mathrm{H}$.

A los 2 años de seguimiento se mantiene en tratamiento con Eculizumab bisemanal, en remisión hematológica y función renal estable: $\mathrm{sCr}$ $1,26 \mathrm{mg} / \mathrm{dL}$ (Figura 1).

\section{Caso 2}

Mujer de 25 años con antecedentes de bulimia, obesidad e hipertensión arterial (HTA). Luego de cirugía bariátrica (a los 24 años) baja $30 \mathrm{~kg}$ de peso y remite la HTA. Usuaria de anticonceptivos orales, fumadora y bebedora de alcohol ocasional.

En controles ambulatorios reaparece la HTA. Se realiza estudio de causa secundaria, destacando: sCr $0,8 \mathrm{mg} / \mathrm{dL}$, orina completa normal, metanefrinas urinarias y pruebas tiroideas normales. Angio-TC descarta alteraciones renales morfológicas y estenosis de arteria renal. Actividad de renina plasmática 22,8 ng/mL/h (normal: 
1,3-4 ng/mL/h) y concentración plasmática de aldosterona $60 \mathrm{ng} / \mathrm{dL}$ (normal: 1,8-23,2 ng/ $\mathrm{dL}$ ). Posteriormente se agrega cuadro de dolor abdominal cólico y distensión que la llevan a consultar. No reporta diarrea, náuseas, vómitos, ni fiebre. Se objetiva HTA $(199 / 120 \mathrm{mmHg})$. Laboratorio: hematocrito $29,9 \%$, hemoglobina $9,7 \mathrm{~g} / \mathrm{dL}$, leucocitos $16.040 / \mu \mathrm{L}$, plaquetas $83.000 /$ $\mu \mathrm{L}$, esquistocitos presentes, $\mathrm{sCr} 2,66 \mathrm{mg} / \mathrm{dL}$, LDH $1.646 \mathrm{UI} / \mathrm{mL}$. Se hospitaliza para estudio y manejo.

Evoluciona con anemización progresiva, trombocitopenia e insuficiencia renal con requerimientos de TRR. Se plantea diagnóstico de MAT. Coombs directo e indirecto (-), ADAMTS13 88\%, $\beta$-HCG negativa, serología VIH, VHC y VHB (-), estudio inmunológico (ANA, ANCA, crioglobulinas, anticuerpos antifosfolípidos, anticoagulante lúpico y anti-estreptolisina $\mathrm{O}$ ) negativos, C’3 81
mg/dL y C'4 26 mg/dL. Proteinuria 9.024 mg/24 h. Se realiza biopsia renal que comprueba la existencia de una MAT.

Inicia metilprednisolona y plasmaféresis. Debido a refractariedad, se plantea tratamiento con Eculizumab. Se administra inmunización para Neisseria meningitidis y Streptococcus pneumoniae. Tras el inicio del fármaco remite el cuadro hematológico. Tres meses más tarde se logra discontinuar HD.

Respecto al estudio genético realizado en el mismo laboratorio del caso previo, se encontró una mutación heterocigota en el gen del Factor $\mathrm{H}$ del complemento (CFH).

Actualmente, luego de 2 años de seguimiento, se mantiene en tratamiento bisemanal con Eculizumab. No ha presentado recaídas de la enfermedad, mantiene remisión hematológica y función renal estable; sCr 1,56 mg/dL (Figura 2).

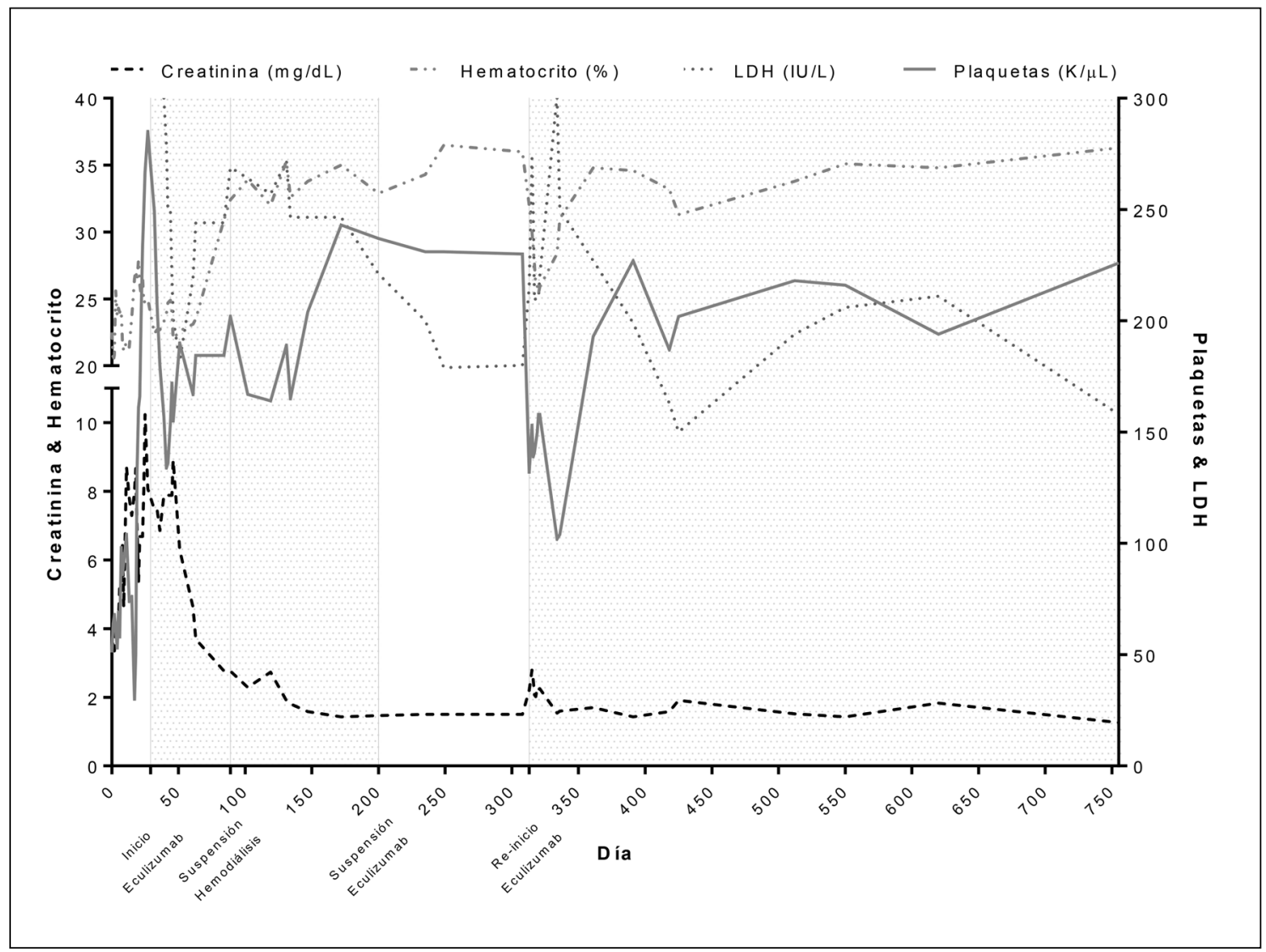

Figura 1. Evolución parámetros asociados a MAT, Caso 1. 


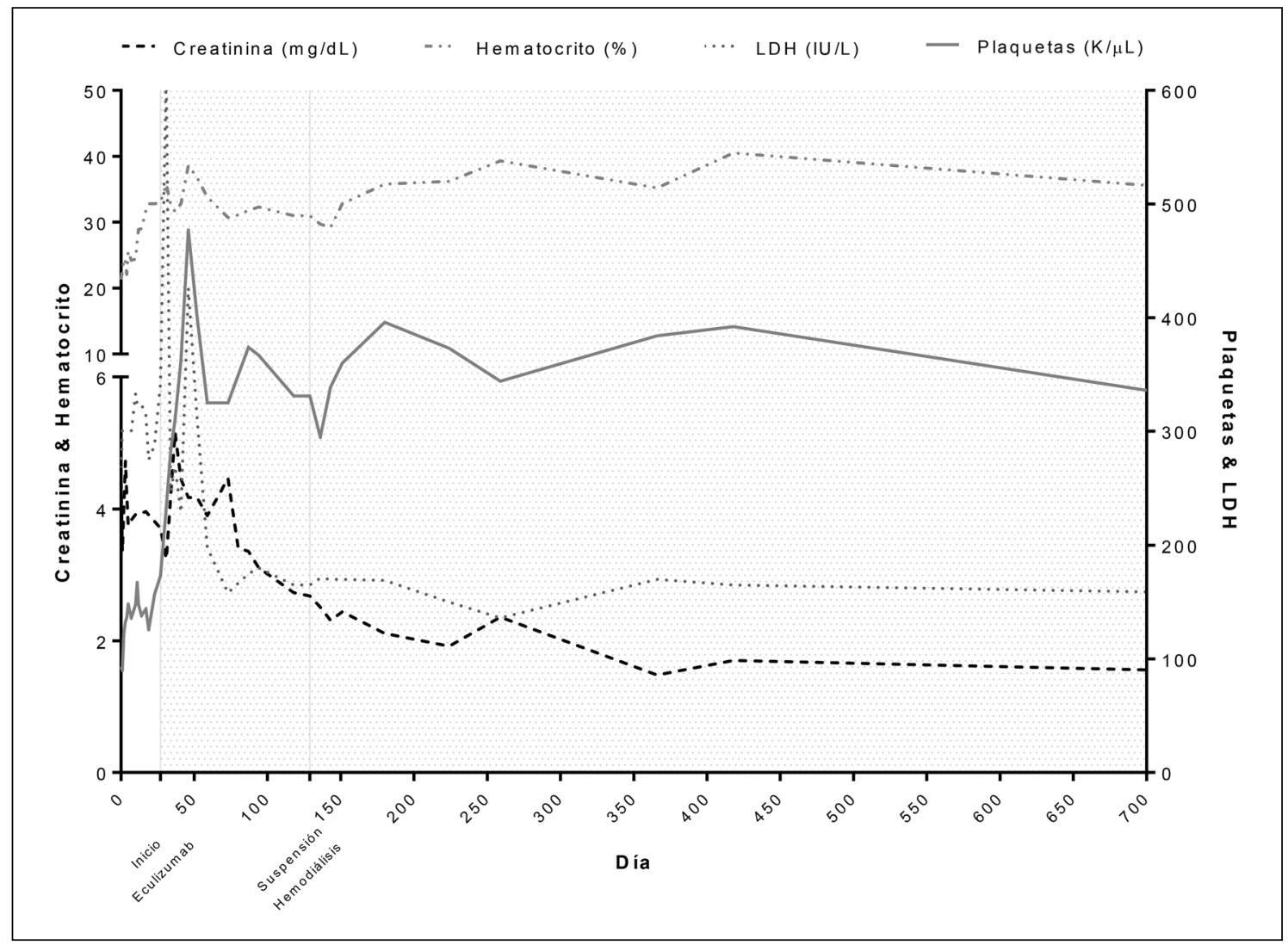

Figura 2. Evolución parámetros asociados a MAT, Caso 2.

\section{Discusión}

Ambas pacientes tienen un cuadro clínico compatible con SHUa ya que presentan MAT (trombocitopenia, anemia hemolítica microangiopática y falla renal) ${ }^{5}$; con exclusión de PTT, SHU típico y MAT secundaria a neoplasias, drogas, infecciones o enfermedades autoinmunes. Estas condiciones son suficientes para asumir SHUa y considerar Eculizumab ${ }^{6}$ (Figura 3).

El cuadro clínico, generalmente es de presentación brusca, pero en $20 \%$ se manifiesta insidiosamente ${ }^{2}$; como en el segundo caso, donde predominó HTA maligna inicial. Se documenta un gatillante en 39-70\% de los SHUa; siendo lo más frecuente cuadros infecciosos gastrointestinales, respiratorios y embarazo/puerperio ${ }^{3,7}$. Entre 23$28 \%$ de los casos presentan una infección intestinal (no asociada a toxina shiga) ${ }^{8}$. En el primer caso, se observa cómo los episodios de SHUa van aso- ciados a infecciones intercurrentes. El embarazo/ puerperio es otro factor gatillante $(20 \%$ de los casos de SHUa en mujeres) $)^{1,5}$; por lo que se descartó en ambas pacientes. El SHUa es propio del post-parto ${ }^{1}$, mientras el PTT ocurre en el segundo y tercer trimestre del embarazo, y es mediado por anticuerpos contra ADAMTS13 ${ }^{5}$.

La afección extrarrenal alcanza 20\% de los casos $^{7}$. Puede comprometer: corazón, intestino, páncreas, pulmones y $\mathrm{SNC}^{3}$. Hasta $30 \%$ presenta diarrea por lo que no es útil para diferenciar con SHU típico ${ }^{6}$. En el primer caso la diarrea pudo corresponder a un evento infeccioso gatillante, $o$ bien, manifestación del SHUa.

El estudio de factores del complemento y anticuerpos, se debe realizar previo a la plasmaféresis. El 36\% de los casos de SHUa tienen C'3 bajo ${ }^{3}$.

Antiguamente se describía el SHUa como una patología propia de población infantil, siendo hoy más prevalente en adulto joven, mayormente mu- 


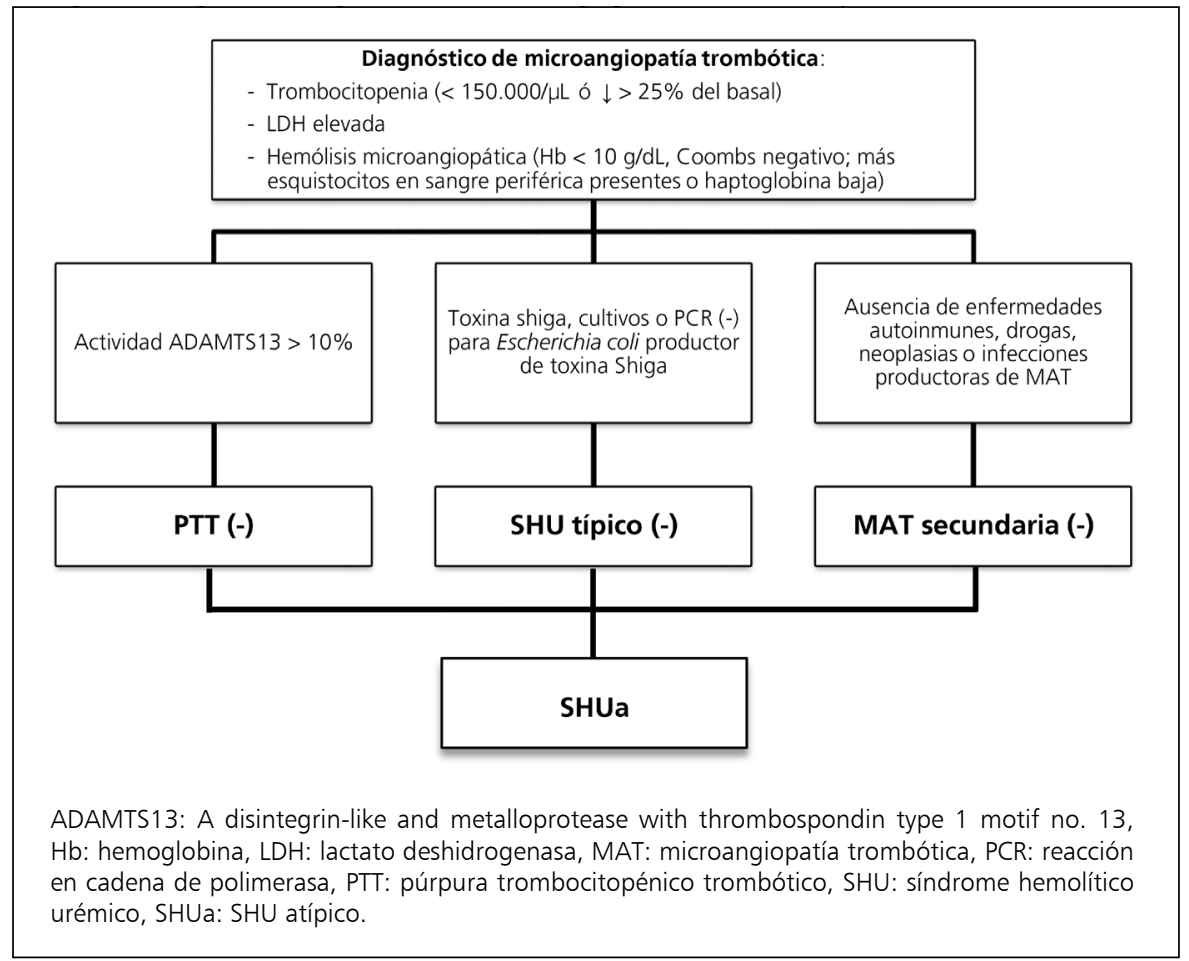

Figura 3. Algoritmo diagnóstico de microangiopatías trombóticas y SHUa. jeres 9 . Se estima que $60-70 \%$ de los casos presenta una alteración genética (SHUa primario) ${ }^{3,6}$.

El pronóstico del SHUa es ominoso. La mortalidad oscila entre $0,8-15 \%$ en el evento agudo ${ }^{7,8}$ y al cabo de 3 a 5 años, 50\% de los infantes y 70\% de los adultos, fallecerán o necesitarán TRR ${ }^{8,10}$. Mutaciones en CFH, CFHR (genes relacionados al $\mathrm{CFH}$ ), C'3 y CFB (factor B del complemento) tienen el peor pronóstico. Al contrario, mutaciones de MCP (proteína cofactor de membrana) tienen múltiples recurrencias, con buen pronóstico y mínima reproducción en trasplante renal ${ }^{7,8}$. En el primer caso se sospechó esta mutación, por la alta recurrencia y recuperabilidad de función renal, sin embargo, se descartó con el estudio genético. Se describe al SHUa sin mutación demostrable como de mejor pronóstico y baja recurrencia ${ }^{10}$. Por este motivo se consideró la suspensión de Eculizumab, sin embargo, la historia de recaídas tuvo un peso mayor, obligando a reinstaurar el tratamiento indefinidamente.

En relación al segundo caso, la mutación del $\mathrm{CFH}$ es la alteración genética más frecuente de
SHUa primario ${ }^{3,6,8}$. El 80\% de los pacientes fallece o está en TRR luego de 5 años, y su recurrencia en trasplante renal es $80 \%{ }^{8,11}$. El $8 \%$ de los casos de SHUa asociados a mutación del CFH son gatillados por embarazo/puerperio o uso de anticonceptivos ${ }^{4}$; situación presente en el caso 2 .

Es fundamental controlar factores que puedan amplificar la actividad del complemento, mediante prevención de infecciones y evitar fármacos de riesgo.

El año 2011 se aprobó Eculizumab para tratamiento del SHUa. Su eficacia está demostrada y permitió cambiar la historia natural de la enferme$\operatorname{dad}^{12,13}$. La inhibición del complemento provoca remisión hematológica, y así, dar pie para que ocurran fenómenos regenerativos con recuperación de la función renal.

En los casos comunicados es necesaria la administración del fármaco en forma indefinida, ya que son pacientes con mutaciones de riesgo o alto grado de recurrencia, y en quienes la terapia ha evitado el desarrollo de enfermedad renal crónica terminal. 


\section{Conclusión}

Se presentan dos casos de SHUa para informar a la comunidad médica sobre esta rara enfermedad: su presentación clínica, pronóstico ominoso, necesidad de diagnóstico oportuno y la posibilidad de cambiar su historia natural gracias al tratamiento con Eculizumab.

\section{Referencias}

1. Loirat C, Frémeaux-Bacchi V. Atypical hemolytic uremic syndrome. Orphanet J Rare Dis 2011; 6 (60): 1-30.

2. Nayer A, Asif A. Atypical Hemolytic-Uremic Syndrome: A Clinical Review. Am J Ther 2016; 23 (1): 151-8.

3. Nester CM, Barbour T, de Cordoba SR, Dragon-Durey MA, Fremeaux-Bacchi V, Goodship TH, et al. Atypical aHUS: State of the art. Mol Immunol 2015; 67 (1): 3142.

4. Noris M, Remuzzi G. Atypical Hemolytic-Uremic Syndrome. N Engl J Med 2009; 361 (17): 1676-87.

5. Riedl M, Fakhouri F, Le Quintrec M, Noone DG, Jungraithmayr TC, Fremeaux-Bacchi, et al. Spectrum of complement-mediated thrombotic microangiopathies: pathogenetic insights identifying novel treatment approaches. Semin Thromb Hemost 2014; 40 (4): 44464.

6. Noris M, Remuzzi G. Glomerular Diseases Dependent on Complement Activation, Including Atypical Hemolytic Uremic Syndrome, Membranoproliferative Glomerulonephritis, and C3 Glomerulopathy: Core Curriculum 2015. Am J Kidney Dis 2015; 66 (2): 359-75.
7. Noris M, Caprioli J, Bresin E, Mossali C, Pianetti G, Gamba S, et al. Relative Role of Genetic Complement Abnormalities in Sporadic and Familial aHUS and Their Impact on Clinical Phenotype. Clin J Am Soc Nephrol 2010; 5 (10): 1844-59.

8. Kavanagh D, Goodship TH. Atypical hemolytic uremic syndrome, genetic basis, and clinical manifestations. Hematology Am Soc Hematol Educ Program 2011; (1): 15-20.

9. Fremeaux-Bacchi V, Fakhouri F, Garnier A, Bienaimé F, Dragon-Durey MA, Ngo S, et al. Genetics and outcome of atypical hemolytic uremic syndrome: a nationwide French series comparing children and adults. Clin J Am Soc Nephrol 2013; 8 (4): 554-62.

10. Fakhouri F, Frémeaux-Bacchi V, Loirat C. Atypical hemolytic uremic syndrome: from the rediscovery of complement to targeted therapy. Eur J Intern Med 2013; 24 (6): 492-5.

11. Zuber J, Le Quintrec M, Morris H, Frémeaux-Bacchi V, Loirat C, Legendre C. Targeted strategies in the prevention and management of atypical HUS recurrence after kidney transplantation. Transplant Rev (Orlando) 2013; 27 (4): 117-25.

12. Legendre CM, Licht C, Muus P, Greenbaum LA, Babu S, Bedrosian C, et al. Terminal complement inhibitor eculizumab in atypical hemolytic-uremic syndrome. N Engl J Med 2013; 368 (23): 2169-81.

13. Licht C, Greenbaum LA, Muus P, Babu S, Bedrosian CL, Cohen DJ, et al. Efficacy and safety of eculizumab in atypical hemolytic uremic syndrome from 2-year extensions of phase 2 studies. Kidney Int 2015; 87 (5): 1061-73. 